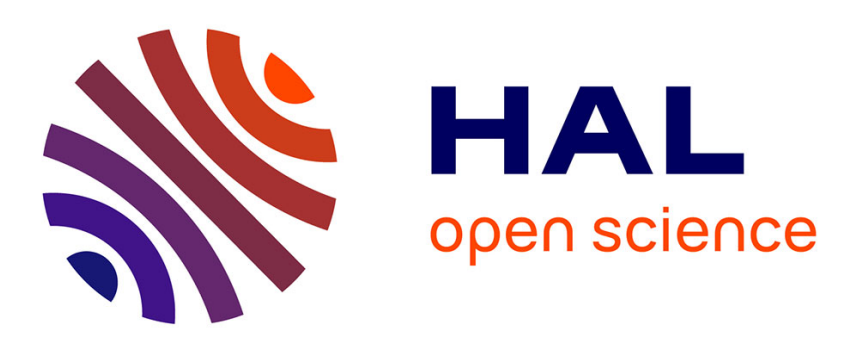

\title{
A coupling phenomenon between the magnetization of two ferromagnetic thin films separated by a thin metallic film-Application to magnetic memories
}

J. Bruyère, O. Massenet, R. Montmory, Louis Néel

\section{- To cite this version:}

J. Bruyère, O. Massenet, R. Montmory, Louis Néel. A coupling phenomenon between the magnetization of two ferromagnetic thin films separated by a thin metallic film-Application to magnetic memories. IEEE Transactions on Magnetics, 1965, 1 (1), pp.10-12. 10.1109/TMAG.1965.1062923 . hal-03214647

\section{HAL Id: hal-03214647 \\ https://hal.science/hal-03214647}

Submitted on 2 May 2021

HAL is a multi-disciplinary open access archive for the deposit and dissemination of scientific research documents, whether they are published or not. The documents may come from teaching and research institutions in France or abroad, or from public or private research centers.
L'archive ouverte pluridisciplinaire HAL, est destinée au dépôt et à la diffusion de documents scientifiques de niveau recherche, publiés ou non, émanant des établissements d'enseignement et de recherche français ou étrangers, des laboratoires publics ou privés. 


\title{
A Coupling Phenomenon Between the Magnetization of Two Ferromagnetic Thin Films Separated by a Thin Metallic Film-Application to Magnetic Memories
}

\author{
J. C. BRUYERE, O. MASSENET, R. MONTMORY, and L. XÉEL
}

\begin{abstract}
A new type of magnetic interaction between two ferromagnetic thin films separated by a third metallic nonmagnetic thin film is studied. This coupling tends to bring the magnetization of the ferromagnetic materials into parallel alignment. The coupling is shown to be dependent upon the intermediate material and its thickness, and on the nature of the ferromagnetic media present. These interactions are observed for intermediate metal thicknesses up to $300 \AA$ in the case of Palladium. The measured surface energy of this coupling is of the order of $0.1 \mathrm{erg} / \mathrm{cm}^{2}$. A study of the variation of the coupling with temperature is given.
\end{abstract}

\section{INTRODUCTION}

$I^{1}$ IS WELL KNOWN [1] that two parallel ferromagnetic films separated by a nonmagnetic film less than $500-\AA$ thick show different types of interactions. The simplest coupling that exists between ferromagnetic thin films is caused by the demagnetizing field which tends to align the magnetization of the ferromagnetic materials in an antiparallel orientation. Fuller and Sullivan [2] and Spain [3], [4] have shown evidence for an interaction between the elementary domain walls. It is also well known that the thin films show an "orange-peel" surface topography. Neel [1] anticipated the existence of magnetostatic interactions when a correlation exists between the interfacial topographies with regard to the ferromagnetic media. The anticipated coupling energy is of the order of $0.01 \mathrm{erg} / \mathrm{cm}^{2}$ for an intermediate layer $100-\AA$ thick and for wavelength irregularities of $400-\AA$ and $40-\AA$ amplitude. Since these three types of interactions are independent of the nature of the intermediate layer, there is no reason why it may not be an insulator.

The new type of coupling described below is observed only if the intermediate material is conducting and if it is less than about $500-\AA$ thick for the films that have been studied.

\section{Experimental Conditions}

The multiple thin films are prepared by vacuum evaporation using a conventional vacuum of $3.10^{-6} \mathrm{~mm}$ of Mercury in the presence of a magnetic field, onto a heated glass substrate. The first layer deposited is a Permalloy type alloy (81 per cent $\mathrm{Ni}, 19$ per cent $\mathrm{Fe}$ ), between $1000-\AA$ and $2000-\AA$ thick, with an uniaxial anisotropy. The top layer is a cobalt-nickel-iron alloy having zero magnetostric-

Manuscript received July 24, 1964. This work was supported by the Centre National de la Recherche Scientifique and the Delegation Generale à la Recherche Scientifique et Technique, Contract 62 FR 163.

The authors are with the Laboratoire d'Electrostatique et de Physique du Métal, C.N.R.S., B.P. 319, Grenoble, France. tion, 1800-A thick with an induced uniaxial anisotropy. These alloys are chosen so that the two materials have different true coercive fields.

Their thicknesses are measured using an oscillating piezoelectric quartz crystal placed in the vacuum chamber [5].

The magnetic characteristics of the multiple films are studied at low frequencies using a special Kerr effect apparatus allowing the hysteresis loops of the two magnetic components of the sandwich to be seen separately on the oscilloscope screen. These measurements are made outside the vacuum chamber.

To study the phenomenon as a function of temperature, the films are placed on a support which can be heated or cooled in another vacuum system. The hysteresis loops are also observed using the Kerr effect at $50 \mathrm{c} / \mathrm{s}$.

\section{Method of Measurement}

The existence of a coupling between two ferromagnetic films is represented by a coupling energy term $E_{c}$. At the same time, the observation of hysteresis loops lead to the introduction of a fictitious coupling field $h$ related to the energy by

$$
E_{c}=J \cdot h \cdot e
$$

where $J$ is the spontaneous magnetization of the observed ferromagnetic film and $e$ is the thickness of this film. The two ferromagnetic media have true coercive fields $H_{c}$ and $H_{c}^{\prime}$ such that $H_{c} \ll H_{c}^{\prime}$.

If the hysteresis loop in the common easy magnetization direction is observed using an alternating field $I I$ with an excursion such that $H>H_{c}{ }^{\prime}$, it can be shown that the apparent coercive fields observed will be

$$
H_{c}+h ; H_{c}^{\prime}-h^{\prime}
$$

$h$ is the fictitious coupling field acting on the medium having a coercive field $H_{c} ; h^{\prime}$ is the fictitious coupling field acting on the medium having a coereive field $H_{c}{ }_{c}$. If $H_{c}$ and $H_{c}{ }^{\prime}$ is known or measured, $h$ and $h^{\prime}$ can be easily determined (see Fig. 1).

\section{Reisults}

\section{Experiments al Room Temperature}

Figure 2 gives the results obtained for intermediate Palladium layers. For thicknesses greater than $300 \AA$, the coupling is no longer measurable. For thicknesses less than $50 \AA$, the value of the coupling field $h$ is such that the 


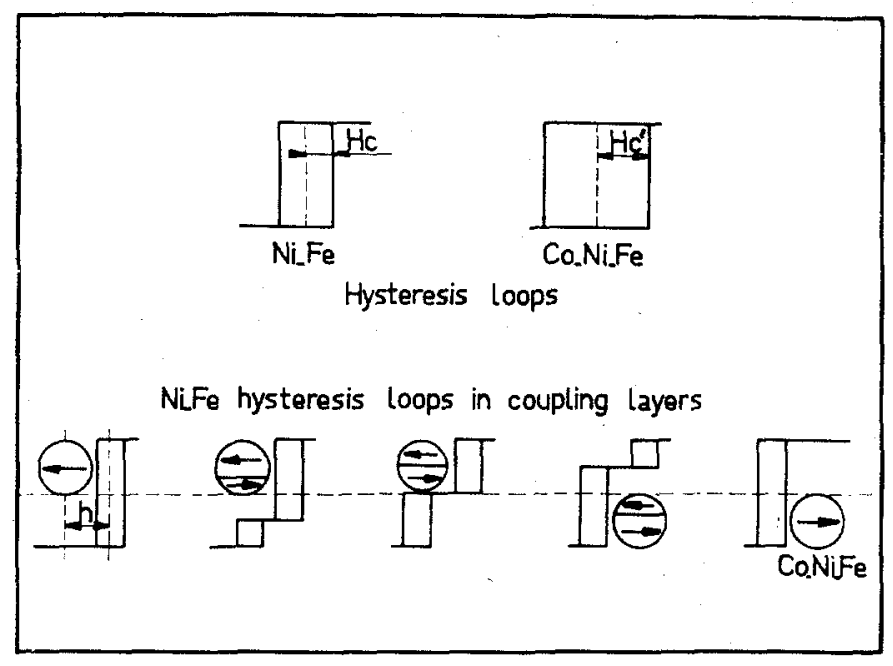

Fig. 1. Different shapes of hysteresis loops for multiple layer coupling.

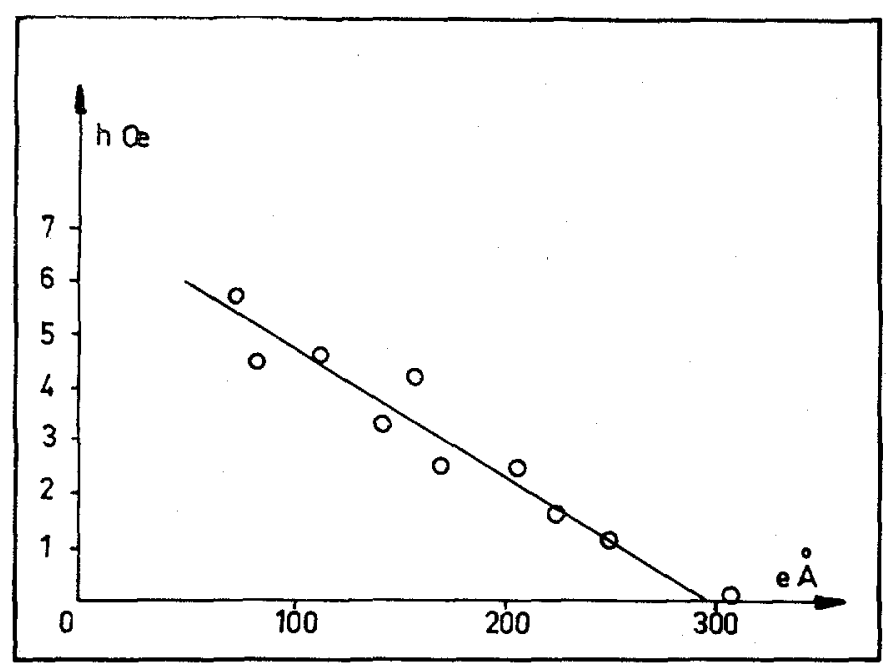

Fig. 2. Coupling field $h$ vs. Palladium layer thickness. Ni-Fe layer of $1800 \AA$. 35 per cent, 52 per cent, 13 per cent $\mathrm{Co}-\mathrm{Ni}$-Fe alloy.

difference between $H_{c}$ and $H_{c}{ }^{\prime}$ is less than $h+h^{\prime}$. In this case, the hysteresis loops for the two magnetic layers in the easy direction are the same. Two distinct loops appear in the hard direction, however, but it is not experimentally possible to obtain a value of the coupling field. Moreover, if the thickness is less than $50 \AA$, there is a possibility of the intermediate layer becoming porous and hence, of quite different phenomena appearing.

Let $J$ be the magnetization of the Ni-Fe layer layer $(J=$ $900 \mathrm{emu}), e$ its thickness $(1800 \AA)$ for $h=6 \mathrm{Oe}$, and let the coupling energy $E_{c}$ be $0.1 \mathrm{erg} / \mathrm{cm}^{2}$, i.e., ten times the maximum energy anticipated by L. Néel [1] for magnetostatic coupling.

The results for intermediate layers of chromium, silver, and gold are summarized in Fig. 3. Several series of experiments have been carried out, especially one using an alloy composed of 45 per cent $\mathrm{Ni}, 45$ per cent $\mathrm{Co}, 10$ per cent $\mathrm{Fe}$, and another using 52 per cent $\mathrm{Ni}, 35$ per cent $\mathrm{Co}$,

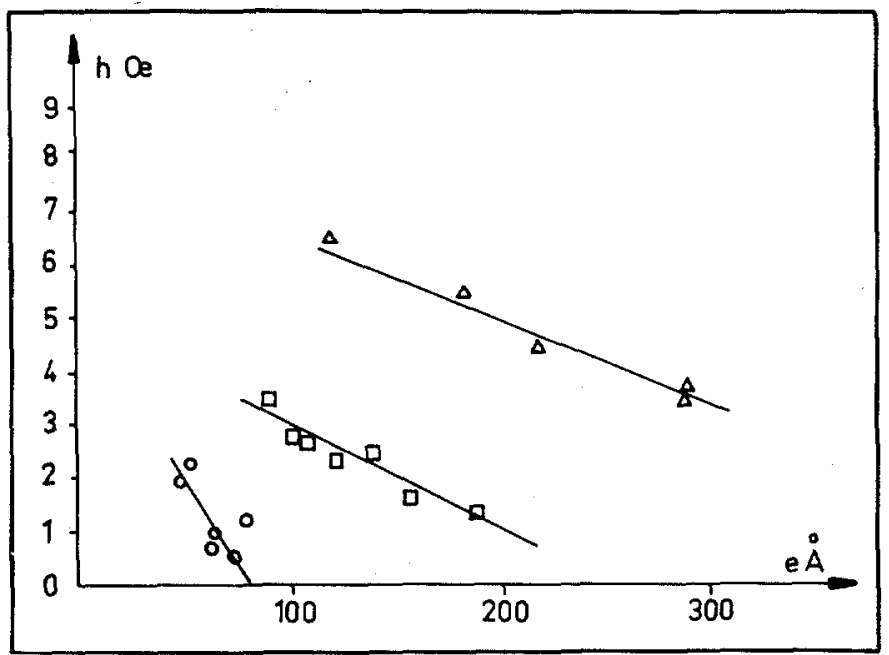

Fig. 3. Coupling field $h$ vs. intermediate layer thickness. G Chromium; $\Delta$ Silver; $O$ Gold. 45 per cent, 45 per cent, 10 per cent Co-Ni-Fe alloy. Ni-Fe layer of $1800 \AA$.

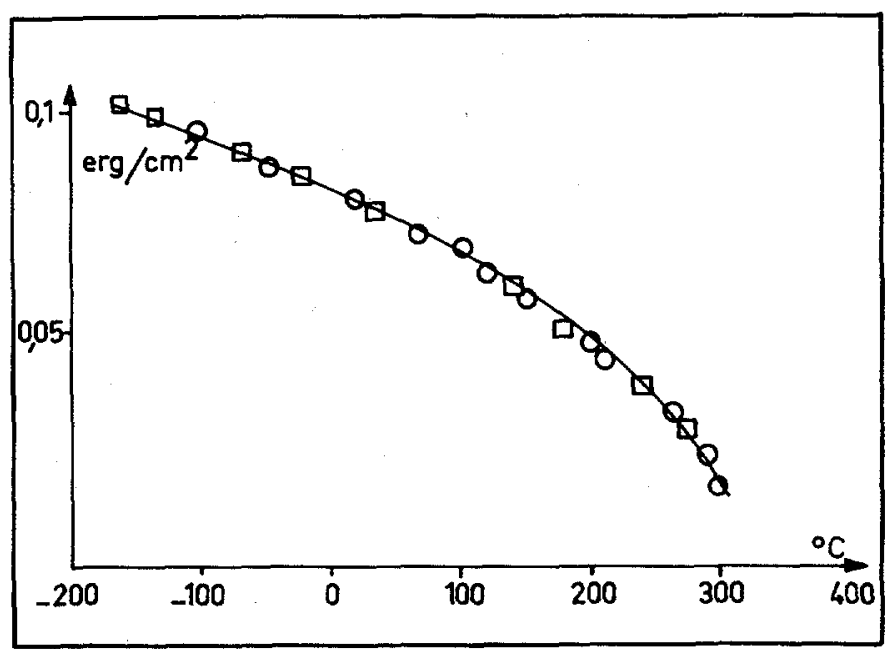

Fig. 4. Coupling energy vs. temperature for a 75 - $\AA$ intermediate layer of Palladium. O heating; $\square$ cooling.

13 per cent Fe [6]. The bulk spontaneous magnetizations of the two alloys are almost the same ( \pm 10 per cent). It is to be noted that for a chromium thickness of $75 \AA$, the coupling strength is three times as great when the composition of the $\mathrm{Co}-\mathrm{Ni}-\mathrm{Fe}$ layer goes from 35 per cent, 52 per cent, 13 per cent to 45 per cent, 45 per cent, 10 per cent. Using gold or silver and an alloy consisting of 35 per cent, 52 per cent, 13 per cent $\mathrm{Co}-\mathrm{Ni}-\mathrm{Fe}$, the coupling fields are weak and hence the results are scattered; the use of an alloy of 45 per cent, 45 per cent, 10 per cent always increases the coupling strength, and the results are more accurate. A linear decrease of the coupling strength with intermediate metal thickness is observed for the following metals: $\mathrm{Au}, \mathrm{Pd}, \mathrm{Cr}, \mathrm{Ag}$, In.

\section{Experiments at Varying Temperatures}

The variation of the coupling energy with temperature for a specimen having an intermediate layer of Palladium 
75- $\AA$ thick is shown in Fig. 4 with the temperature varying continuously from $-180^{\circ} \mathrm{C}$ to $300^{\circ} \mathrm{C}$. A significant decrease of coupling energy with rise in temperature is observed. Similar experiments carried out using samples with intermediate layers of $\mathrm{Cr}, \mathrm{Ag}, \mathrm{Au}$ show a much slower variation with temperature.

It has also been observed that, for a sample showing no coupling phenomena at room temperature (the Palladium layer was thick: > $300 \AA$ ), an appreciable coupling field appeared on cooling. At $-180^{\circ}$ the measured coupling field was $1 \mathrm{Oe}$. There is no thermal hysteresis.

The observation of the rapid variation of coupling energy with temperature for Pd suggests that this phenomenon cannot be of a magnetostatic origin. In fact if it had been, it could only be explained by a decrease of the spontaneous magnetization or by a reversible modification of the layer structure.

\section{Application to Magnetric Memories}

A memory element consisting of a "reading" layer with a weak anisotropy field separated from a "memory" layer with a higher anisotropy field by an appropriate metallic layer can be made. After recording the information, a magnetic field pulse applied in the hard direction turns the "reading" layer magnetization perpendicular to the easy direction, while the "memory" layer magnetization is turned through less than $90^{\circ}$. At the end of the pulse, the memory layer magnetization returns spontaneously to its original position, bringing with it the "reading" layer magnetization, because the two magnetizations are coupled.

The interest of this type of memory element lies in its novel construction and use. In fact, the coupling energy can easily be adjusted to the desired value by changing the nature and thickness of the intermediate layer material. Finally, the fact that there is a "positive" coupling (that is, one which tends to bring the magnetizations of the two films parallel) allows the strip lines to be placed outside the memory element.

\section{REFERFNCES}

[1] Néel, L. CaR Acad Sci: (Paris), vol 255, 1962, pp 1676-1681. [2] Fuller, H. W., and D. L. Sullivan, J. A ppl: Phys., vol 33, 1962, pp. 1063-1064

[3] Spain, R. J., and I. B. Puchalska, C.R. Acad. Sci. (Paris), vol 254,1962 , pp 2987-2989.

[4] Spain, R. J., Ph. D. dissertation, Université de Paris, France, 1963 , pp 79-85.

[5] Bruyere, J. C., and R. Billat, French Patent CNRS P.Y. 957.650.

[6] Bradley, E. M., J. Appl. Phys., vol 33, 1962, pp 1051-1957.

[7] Néel, L., J. C. Bruyère, $O$. Massenet, and R. Montmory, French Patent CNRS P.V.951.108. 